\title{
EFECTO DE LA PROFUNDIDAD DEL SUELO EN Rottboellia cochinchinensis (LOUR) CLAYTON EN CAÑA DE AZÚCAR (Saccharum officinarum L.) ${ }^{1}$
}

\author{
Ramón León ${ }^{2}$, Renán Agüero ${ }^{3}$
}

\section{RESUMEN}

Efecto de la profundidad del suelo en Rottboellia cochinchinensis (Lour) Clayton en caña de azúcar (Saccharum officinarum L.). Se muestrearon cuatro lotes con diferente textura de suelo, sembrados con caña de azúcar durante cuatro años consecutivos. El lote A con suelo franco arcilloso, el lote $\mathrm{B}$ con suelo franco arenoso, el lote $\mathrm{C}$ con suelo arcilloso y el lote D con suelo franco. Se seleccionaron lotes con una población de $R$. cochinchinensis de 75 a 150 plantas $/ \mathrm{m}^{2}$. Se contó el número de plantas de la maleza en un área de $0,25 \mathrm{~m}^{2}$, en dos micro-hábitats: el surco y entre-surco. A cada planta se le escarbó el suelo que la rodeaba hasta encontrar la semilla de la que provenía y se midió la profundidad a la que ésta se encontró, para luego determinar qué porcentaje del total de plantas contabilizadas provenía de semillas localizadas en cada uno de los siguientes estratos: 0,$0 ;>0,0$ a 2,5; $>2,5$ a 5,$0 ;>5,0$ a 10 y $>10,0$ a $15,0 \mathrm{~cm}$ de profundidad. Además, se evaluó en invernadero el efecto de cada profundidad de los estratos citados. A nivel de laboratorio y en platos Petri, se evaluó el efecto de la luz y la oscuridad sobre la germinación de la semilla de $R$. cochinchinensis. Se encontró que la mayoría de las plantas en campo provenían de semillas que se encontraban de $>0$ a $2,5 \mathrm{~cm}$, seguido por aquellas que estaban de $>2,5$ a $5,0 \mathrm{~cm}$ donde se dieron las mejores condiciones para la germinación. No se observaron diferencias entre los tipos de suelo o los micro-hábitats evaluados. En invernadero el tratamiento a $0,0 \mathrm{~cm}$ fue el que mostró la mayor germinación y esto fue producto de la exposición a la luz, lo cual se corroboró en el estudio en laboratorio donde el tratamiento con luz presentó también, la mayor germinación.

\begin{abstract}
Effect of soil depth on the establishment of plants of Rottboellia cochinchinensis (Lour) Clayton in sugar cane (Saccharum officinarum L.). Four sugarcane fields with different soil textures (clay loam, sandy loam, clay and loam) were sampled. All fields showed $R$. cochinchinensis populations with at least $75-150$ plants $/ \mathrm{m}^{2}$. The number of plants of this weed in $0.25 \mathrm{~m}^{2}$ was determined separately in the microhabitats: rows and between rows. The depth at which each plant emerged was measured. Based on depth emergence, each plant was distributed in one of the next categories: $0,>0-2.5,>2.5-5.0,>5.0-10$ and $>10.0-15.0 \mathrm{~cm}$. In addition, under greenhouse conditions, seeds were seeded in pots at the maximum depth of each category in order to evaluate the effect of soil depth. Finally, the effect of light and darkness on seed germination were evaluated in Petri dishes under laboratory conditions. In the field evaluations most of the plants emerged from $>0-2.5 \mathrm{~cm}$. The second most common category was $>2.5-5.0 \mathrm{~cm}$. It seems that those depths provide optimum conditions for germination. There were no differences regarding soil texture or microhabitats. In the greenhouse experiment, the largest germination was observed at $0 \mathrm{~cm}$ due to light exposure in this treatment. This was confirmed in the laboratory where light treatment showed also the largest germination. Control strategies that keep the seeds of this species above ground will reduce the number of new seeds in the seed bank and avoid optimum field conditions for its germination. If such strategies include efforts to reduce the seed production of those plants that are able to become established, it could be possible to significantly reduce the seed bank, therefore, it would be easier to control the population of this weed.
\end{abstract}

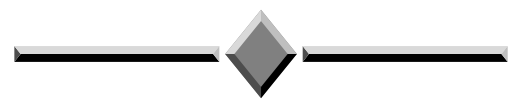

\section{INTRODUCCIÓN}

El uso racional de los recursos es un tema cada vez más cotidiano en todos los ámbitos y los sistemas agrícolas no escapan a ello. Se debe procurar obtener el mayor provecho de los elementos o sistemas con que se cuente y al mínimo costo posible. Esto quiere decir que el detrimento que sufren los recursos por su explotación sea el menor.

En muchos agroecosistemas el manejo integrado de malezas es de suma importancia para el rendimiento

\footnotetext{
1 Proyecto de Investigación, Laboratorio de Malezas, CIPROC. Universidad de Costa Rica.

2 Agronomy Department Iowa State University, Ames, IA 50011. E-mail: rleon@iastate.edu.

3 CIPROC. Facultad de Agronomía, Universidad de Costa Rica. E-mail: raguero@cariari.ucr.ac.cr
} 
del cultivo y más importante aún, para la rentabilidad del proceso productivo. Este tipo de manejo incluye todas las herramientas y tácticas con las que pueda contar un productor, ya sean culturales, físicas, químicas o biológicas, entre otras. El objetivo no es seleccionar una o dos de éstas, sino utilizarlas todas haciendo un uso racional, de tal manera que se complementen mutuamente, regulando las poblaciones de las malezas en forma eficiente, barata, equilibrada, sostenida y ocasionando el menor impacto ambiental (Zimdahl, 1995).

Una táctica de control de poblaciones de malezas en los agroecosistemas, ampliamente utilizada por muchos años, es la labranza del suelo. Esta es de suma importancia, ya que no solamente determina el comportamiento de la población de malezas presentes en un momento dado, o ciclo productivo, sino que influye en el banco de semillas, por lo que puede determinar el comportamiento de poblaciones futuras (Hartzler y Buhler, 1998). Dependiendo de qué tipo de labranza y con qué equipo se realice, se favorecerá una distribución homogénea de la cantidad y calidad de semilla de malezas en el perfil del suelo, como en el caso de la labranza con discos, o por el contrario se favorecerá una concentración en algún estrato definido del perfil como ocurre con la mínima labranza o la labranza cero (Buhler et al., 1997; Hoffman et al., 1998).

Rottboellia cochinchinensis es una de las malezas más problemáticas en muchos cultivos, como maíz, algodón, arroz de secano, frijol, soya, caña de azúcar, etc. (Patterson et al, 1979; Hernández y Soto, 1989; Herrera, 1989; Lencse y Griffin, 1991; Millhollon y Burner, 1993; Calvo et al, 1996; Merayo et al, 1998; Bolaños y Alfaro, 1999), y la mayoría de éstos se producen con un alto nivel de mecanización, especialmente de labranza del suelo. Esta maleza puede producir más de 15000 semillas por planta durante su ciclo de vida (Rojas et al, 1994), las cuales si son enterradas, mantienen su viabilidad por un período de hasta tres o cuatro años, al final del cual, el porcentaje de semillas que mantiene su capacidad germinativa es sumamente bajo. Además, se ha observado que si las condiciones de humedad y temperatura son adecuadas y la semilla no se entierra, se pueden producir hasta cuatro generaciones consecutivas en menos de dos meses. Debido a la importancia de la presencia de semillas de $R$. cochinchinensis dentro del manejo de muchos cultivos es que es indispensable desarrollar estrategias que ayuden a colocarlas en lugares o condiciones que le dificulten su germinación y desarrollo y que ésto consecuentemente de como resultado una población menor que será más fácilmente regulable por medio de otras herramientas o estrategias (Griffin, 1991; Millhollon, 1993).
El objetivo de este trabajo fue determinar si la profundidad a la que se puede encontrar la semilla de $R$. cochinchinensis en el suelo tiene algún efecto sobre el establecimiento de sus plántulas.

\section{MATERIALES Y MÉTODOS}

\section{Determinación de la profundidad de emergen- cia de las plántulas}

El estudio se realizó en el cantón de Cañas, en la provincia de Guanacaste, en Costa Rica. Según Holdridge (1982) esta zona se clasifica como Bosque Seco Tropical y presenta dos períodos climáticos bien definidos, uno lluvioso que se presenta desde mediados de mayo hasta mediados de noviembre y uno seco muy estable, desde mediados de noviembre hasta mediados de mayo. Esta región se encuentra entre 0 y 100 msnm, presenta una temperatura promedio de $27,5^{\circ} \mathrm{C}$, con mínima de $22,7^{\circ} \mathrm{C}$ y máxima de $32,4^{\circ} \mathrm{C}$. La humedad relativa va de $82 \%$ como máxima, a $54 \%$ como mínima con media de $66 \%$ y la precipitación promedio anual es de $1500-1800 \mathrm{~mm}$, la cual se distribuye en forma errática e irregular.

Se muestrearon cuatro lotes con diferente textura de suelo, que han permanecido sembrados con caña de azúcar durante al menos cuatro años consecutivos. El lote A con suelo franco arcilloso, el lote B con suelo franco arenoso, el lote $\mathrm{C}$ con suelo arcilloso y el lote $\mathrm{D}$ con suelo franco. Los lotes seleccionados tuvieron una población de $R$. cochinchinensis de 75 a 150 plantas $/ \mathrm{m}^{2}$. Los muestreos se realizaron de tres a cuatro semanas después del primer riego para que la mayoría de las plantas presentaran al menos entre dos y tres hojas.

Se muestrearon 10 sitios distribuidos al azar en cada lote en dos microhábitats: el surco y el entre surco. El primero consiste en la línea de siembra de la caña de azúcar y el segundo la zona que se encuentra entre dos surcos contiguos. En cada punto, se contó el número de plantas de la maleza que habían expandido completamente al menos su primera hoja y estaban emitiendo la segunda, y que se encontraron en un área de $0,25 \mathrm{~m}^{2}$, que se determinó con ayuda de un marco de $0,5 \mathrm{~m}$ de lado. Luego a cada planta se le escarbó el suelo que la rodeaba hasta encontrar la semilla de la que provenía (ya que esta permanece adherida a la raíz) y se medió la profundidad a la que esta se encontró. Luego se determinó qué porcentaje del total de plantas contabilizadas provenía de semillas localizadas, en cada uno de los siguientes seis estratos: 
I. $\quad 0,0 \mathrm{~m}$ (sobre la superficie)

II. $>0,0$ a $0,025 \mathrm{~m}$

III. $>0,025$ a $0,05 \mathrm{~m}$

IV. $>0,050$ a $0,10 \mathrm{~m}$

V. $>0,10$ a $0,15 \mathrm{~m}$

\section{Aislamiento del efecto de la profundidad}

Se colectó semilla de la maleza, directamente de las espigas de sus individuos. Esta se mantuvo en oscuridad por 30 días. Luego se procedió a establecer seis tratamientos que consistieron en sembrar 50 semillas en potes con una capacidad de 3,785 litros, a cada una de las máximas profundidades correspondientes a los estratos de los estudios anteriores. Los potes se colocaron en un invernadero y se mantuvieron a capacidad de campo con riego por capilaridad durante un mes y al finalizar éste, se contaron las plantas establecidas, según el criterio antes citado. Se utilizó un diseño experimental irrestricto al azar con seis repeticiones.

\section{Efecto de la luz sobre la germinación}

Para evaluar la importancia de la luz sobre la germinación, bajo condiciones de laboratorio, se establecieron dos tratamientos, uno donde la semilla se expuso a la luz y otro donde no se dio esta condición. Para esto, con el mismo material colectado para el estudio en invernadero se colocaron 25 semillas en platos petri los cuales poseían un papel de filtro húmedo. Para el tratamiento sin luz se cubrió el plato petri con papel aluminio de tal forma que las semillas quedaran en oscuridad total y en el caso del tratamiento con luz el plato petri no se cubrió. Los tratamientos se colocaron a luz solar normal, en períodos de 12 horas luz y 12 oscuridad en el caso del tratamiento con luz. Esto se realizó por un total de 30 días. Se utilizó un diseño irrestricto al azar con cuatro repeticiones. $\mathrm{Al}$ final del experimento se determinó el número de semillas que presentaron una radícula de más de $2 \mathrm{~mm}$, a las que se consideró germinadas.

Los datos obtenidos en los diferentes estudios se transformaron a porcentaje para ser analizados. Los resultados se analizaron mediante análisis de variancia $(\mathrm{P}<0,05)$ y para hacer separación de medias se utilizó la prueba de la Diferencia Mínima Significativa $(\mathrm{P}<0,05)$.

\section{RESULTADOS Y DISCUSIÓN}

Como se aprecia en la Figura 1, la mayoría de las plantas provenían de semillas que se encontraron en los

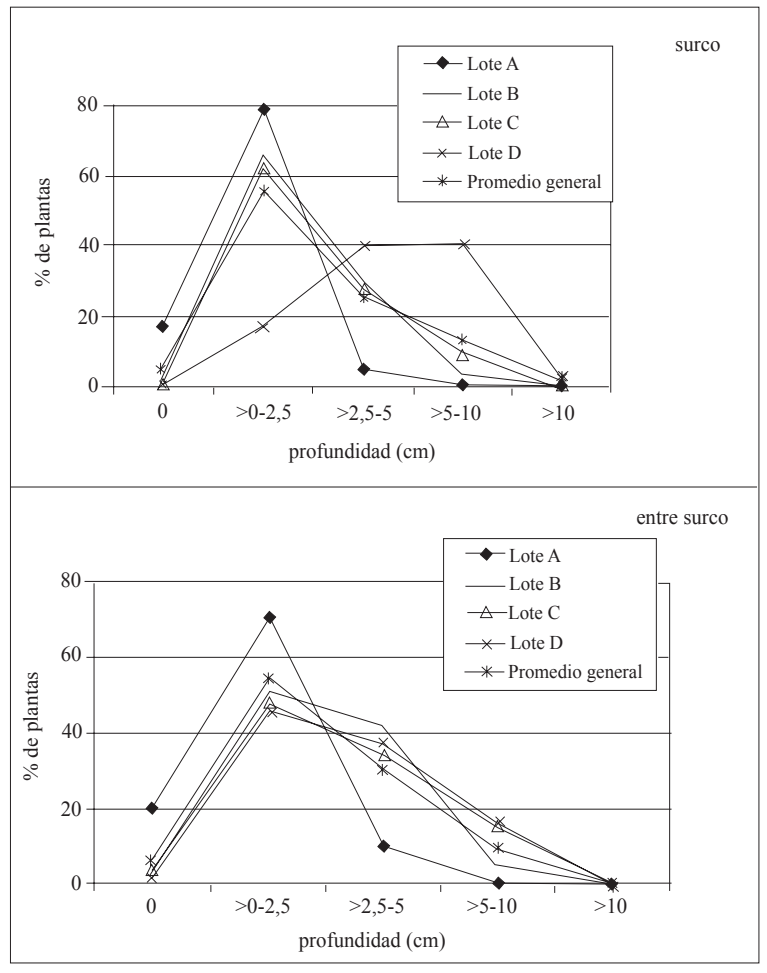

Figura 1. Porcentaje de plantas de Rottboellia cochinchinensis (Lour.) Clayton según el estrato de profundidad en el cual se encontraba la semilla de la cual emergieron, en cuatro lotes sembrados con caña de azúcar, en el Trópico seco de Costa Rica.

primeros $5 \mathrm{~cm}$ de profundidad, en el caso del surco fue el $86 \%$ y del entre surco el $91 \%$. En ambos micro-hábitats, cerca del $55 \%$ de las plantas emergieron del estrato II y solamente poco más del 5\% lo hizo del estrato I. El estrato IV mostró porcentajes similares al I y del estrato $\mathrm{V}$ prácticamente no emergieron plantas.

Al analizar los resultados con respecto al tipo de suelo, prácticamente no hubo diferencias en el comportamiento observado, lo mismo que para el surco y el entre surco. Solamente en el lote D, cuya textura de suelo era franca, se observó que en el surco, el $80 \%$ de las plantas provenían de semillas que estaban en los estratos III y IV y que cada uno representó el $40 \%$.

En el experimento en invernadero, el mayor porcentaje de emergencia se obtuvo en el estrato I (Cuadro 1), el cual mostró $41,7 \%$, mientras que los demás estratos no superaron el $1,7 \%$ y no difirieron entre ellos.

Por su parte, los resultados obtenidos en el estudio de germinación en platos petri, permitieron observar que la luz directa sobre la semilla de $R$. cochinchinensis estimula la germinación, ya que como se ve en el 
Cuadro 1. Porcentaje de plantas de Rottboellia cochinchinensis (Lour.) Clayton según el estrato de profundidad en el cual se encontraba la semilla de la cual emergieron, en un ensayo con potes en invernadero.

\begin{tabular}{llc}
\hline \multicolumn{2}{l}{ Estrato } & Porcentaje de plantas emergidas \\
\hline I & $(0.0 \mathrm{~cm})$ & $41,7 \mathrm{~b}^{*}$ \\
II & $(>0,0$ a $2,5 \mathrm{~cm})$ & $0,7 \mathrm{a}$ \\
III & $(>2,5$ a $5,0 \mathrm{~cm})$ & $1,7 \mathrm{a}$ \\
IV & $(>5,0$ a $10,0 \mathrm{~cm})$ & $0,3 \mathrm{a}$ \\
V & $(>10,0$ a $15,0 \mathrm{~cm})$ & $0 \mathrm{a}$ \\
\hline
\end{tabular}

* Separación de medias según la prueba D.M.S. $(\mathrm{P}<0,05)$.

Cuadro 2, el tratamiento con luz fue significativamente superior al de oscuridad.

Rojas et al. (1994) observaron en un estudio realizado en el trópico seco de Costa Rica, que las mejores condiciones para la germinación de esta especie se dieron a cinco $\mathrm{cm}$ de profundidad, además la menor germinación se dio a $0 \mathrm{~cm}$ y a $20 \mathrm{~cm}$. Sin embargo, encontraron que sobre la superficie del suelo la persistencia (viabilidad en el tiempo) de la semilla fue muy baja en la superficie, mientras que a 10 ó $20 \mathrm{~cm}$ de profundidad la persistencia fue mucho mayor. En un estudio similar, Bridgemohan et al. (1991) observaron que la mayor germinación se dio en los primeros $5 \mathrm{~cm}$, siendo mayor a los $0 \mathrm{~cm}$, además encontraron que la principal causa de mortalidad de semillas fue la pudrición, la cual se debió en gran parte a la acción de hongos.

Cuadro 2. Porcentaje de germinación en platos Petri de semillas de Rottboellia cochinchinensis (Lour.) Clayton bajo dos condiciones: luz y oscuridad.

\begin{tabular}{lc}
\hline Tratamiento & Porcentaje de semillas germinadas \\
\hline Luz & $19 \mathrm{~b}^{*}$ \\
Oscuridad & $6 \mathrm{a}$ \\
\hline
\end{tabular}

*Separación de medias según la prueba D.M.S. $(\mathrm{P}<0,05)$.

La germinación de semillas de esta maleza sobre la superficie del suelo es un comportamiento que se ha reportado tanto en condiciones controladas como de campo (Hérnandez y Soto, 1990; Merayo et al., 1998). Uno de los principales factores que estimulan la germinación de la semilla sin que ésta sea enterrada es la incidencia directa de luz ya que rompe su latencia (Pamplona y Mercado, 1974; Hérnandez y Soto, 1990), aunque Jantawinurag y Suwanketnikom (1996) no encontraron respuesta a este factor. Otros factores que son de importancia cuando la semilla está en la superficie del suelo o a poca profundidad es la alternancia de humedecimiento y secado y el contacto con las partículas del suelo ya que tienen un efecto estimulante sobre la germinación (Thomas y Allison, 1975). Esto explica los resultados obtenidos en invernadero y laboratorio, donde los tratamientos que mostraron la mayor emergencia y germinación, respectivamente, fueron aquellos que recibieron luz.

Si bien en los primeros cinco $\mathrm{cm}$ de profundidad del suelo se dan las mejores condiciones para la germinación de las semillas de $R$. cochinchinensis, prácticas que busquen profundizar dicha semilla no son las más adecuadas ya que tal acción favorece la persistencia de estas en el tiempo (Bridgemohan et al, 1991; Rojas et $a l, 1994)$. Una estrategia de manejo más acertada sería procurar que la semilla permaneciera sobre la superficie donde, los factores ambientales y biológicos, fomenten la pérdida de viabilidad y la mantengan en un estrato donde las condiciones no son las óptimas para la germinación, como sí ocurre en los estratos II y III del presente trabajo. En este sentido la cero labranza en conjunto con métodos de control que disminuyan al mínimo la producción de semillas por parte de las plantas que logren establecerse, puede ser una de las mejores formas de controlar a esta especie y que en circunstancias donde el banco de semillas sea grande se propicie en forma efectiva la disminución de éste, en un lapso corto de tiempo, ya que la viabilidad de esta especie varía entre los tres a cuatro años (Thomas, 1973; Bridgemohan y Brathwalte, 1989).

\section{LITERATURA CITADA}

BOLAÑOS, J.; ALFARO, R. 1999. Diagnóstico preliminar sobre la distribución y propagación de Rottboellia cochinchinensis (Lour.) W. D. Clayton en las plantaciones de caña de azúcar en Costa Rica. In Memorias XI Congreso Nacional Agronómico y de Recursos Naturales. Volumen II: Manejo de Cultivos. 196 p.

BRIDGEMOHAN, P.; BRATHWAITE, R. 1989. Weed management strategies for the control of $R$. cochinchinensis in maize in Trinidad. Weed Research 29: 433-440.

BRIDGEMOHAN, P.; BRATHWAITE, R.; MACDAVID, C. 1991. Seed survival and patterns of seedling emergence studies of Rottboellia cochinchinensis (Lour.) W. D. Clayton in cultivated soils. Weed Research 31: 265-272.

BUHLER, D.; HARTZLER, R.; FORCELLA, F. 1997. Implications of weed seedbank dynamics to weed management. Weed Science 45: 329-336. 
CALVO, G.; MERAYO, A.; ROJAS, E. 1996. Diagnóstico de la problemática de la caminadora (Rottboellia cochinchinensis) en dos zonas productoras de maíz de la provincia de Guanacaste, Costa Rica. Manejo Integrado de Plagas 41: 50-52.

GRIFFIN, J. 1991. Itchgrass (Rottboellia cochinchinensis) control options in soybean (Glicine max). Weed Technology 5: 426-429.

HARTZLER, R.; BUHLER, D. 1998. Weed seeds and the seedbank: implications for weed management. Iowa State University. University Extention. 6 p.

HERNÁNDEZ, R.; SOTO, A. 1989. Influencia del régimen de humedad en el suelo sobre el crecimiento de Rottboellia exaltata en invernadero. Agronomía Costarricense 13(2): 203-210.

HERNÁNDEZ, R.; SOTO, A. 1990. Influencia del déficit hídrico en la germinación de Rottboellia exaltata bajo sequía simulada. Agronomía Costarricense 14(2): 135140.

HERRERA, F. 1989. Situación de Rottboellia cochinchinensis en Costa Rica. In: Seminario Taller sobre "Rottboellia cochichinensis Lour.”y “Cyperus rotundus L.”, distribución, problemas e impacto económico en Centroamérica y Panamá. Proyecto MIP-CATIE, Honduras. p. 14.

HOFFMAN, M.; OWEN, M.; BUHLER, D. 1998. Effects of crop and weed management on density and vertical distribution of weed seeds in soil. Agronomy Journal 90: 793-799.

HOLDRIDGE, L. 1982. Ecología basada en zonas de vida. 1a. ed. , 2a. reimpresión. San José, Costa Rica. IICA. $216 \mathrm{p}$.

JANTAWINYURAG, R.; SUWANKETNIKOM, R. 1996. Factors influencing seed germination of itchgrass. Kasetsart Journal 30: 419-428.
LENCSE, R.; GRIFFIN, J. 1991. Itchgrass (Rottboellia cochinchinensis) interference in sugarcane (Saccharum sp.). Weed Technology 5: 396-399.

MERAYO, A.; ROJAS, C.; VALVERDE, B.; UMAÑA, E. 1998. Leguminosas de cobertura para el manejo de Rottboellia cochinchinensis en el asocio yuca/maíz. Manejo Integrado de Plagas 48: 49-53

MILLHOLLON, R. 1993. Preemergence control of Itchgrass (Rottboellia cochinchinensis) and Johnsongrass (Sorghum halepense) in sugarcane (Saccharum spp. Hybrids) with Pendimethalin and Prodiamine. Weed Science. 41: 621-626.

MILLHOLLON, R.; BURNER, D. 1993. Itchgrass (Rottboellia cochinchinensis) biotypes in world populations. Weed Science 41: 379-387.

PAMPLONA, P.; MERCADO, B. 1974. Methods of controlling Rottboellia exaltata in corn. Philipine Weed Science Bulletin 4: 13-20.

PATTERSON, D.; MEYER, C.; FLINT, E.; QUIMBY, P. 1979. Temperature responses and potential distribution of itchgrass (Rottboellia exaltata) in the United States. Weed Science 27(1): 77-82.

ROJAS, E.; MERAYO, A.; CALVO, G. 1994. La profundidad y duración en el suelo de la semilla de caminadora (Rottboellia cochinchienensis (Lour.) W. D. Clayton) y su efecto sobre la viabilidad y persistencia en el Trópico Seco. Manejo Integrado de Plagas 32: 25-29.

THOMAS, P. 1973. Studies on Rottboellia exaltata (Shamva grass or guinea fowl grass). Rhodesia Agricultural Journal 6: 140-142.

THOMAS, P.; ALLISON, J. 1975. Seed dormancy and germination in $R$. exaltata. Journal of Agricultural Science, Cambridge 85: 12-134.

ZIMDAHL, R. 1995. Weed science in sustainable agriculture. American Journal of Alternative Agriculture 10(3): 138-142. 